\title{
Activities of daily living with reverse prostheses: importance of scapular compensation for functional mobility of the shoulder
}

\author{
Alexandre Terrier, $\mathrm{PhD}^{\mathrm{a}, *}$, Patricia Scheuber, $\mathrm{MSc}^{\mathrm{a}}$, Dominique P. Pioletti, PhD ${ }^{\mathrm{a}}$, \\ Alain Farron, $M D^{b}$
}

${ }^{a}$ Laboratory of Biomechanical Orthopedics, Ecole Polytechnique Fédérale de Lausanne (EPFL), Lausanne, Switzerland

${ }^{b}$ Service of Orthopaedics and Traumatology, University Hospital Center and University of Lausanne (CHUV), Lausanne, Switzerland

\begin{abstract}
Hypothesis: The nonanatomical design of reverse shoulder prostheses induce medial displacement of the center of rotation, impingements and may reduce the mobility of the shoulder. The aim of this study is to test the hypothesis that during activities of daily living functional mobility of the shoulder can be restored by scapular compensation.

Material and methods: A numerical 3-dimensional model was developed to reproduce the movement of the scapula and humerus, during 4 activities of daily living measured experimentally. This hypothesis was tested in 4 configurations of the aequalis reverse prosthesis (standard 36-mm glenosphere, 42-mm glenosphere, lateralized 36-mm glenosphere, lateralized Bony Increased-Offset Reverse Shoulder Arthroplasty [BIO-RSA]), which were implanted in the virtual model. All impingement positions were evaluated, as the required scapular compensation to avoid impingements.

Results: With the 36-mm glenosphere, impingements occurred only for rest of hand to back-pocket positions. The 42-mm partly improved the mobility. The 2 lateralized glenospheres were free of impingement. When impingements occurred, the scapular compensation was less than $10^{\circ}$.

Conclusion: Most reverse prostheses impingements reported in clinical and biomechanical studies can be avoided, either by scapular compensation or by a glenosphere lateralization. After reverse shoulder arthroplasty, a fraction of the mobility of the gleno-humeral is transferred to the scapulo-thoracic joint. Level of evidence: Basic Science Study, Computer Modeling.

(C) 2013 Journal of Shoulder and Elbow Surgery Board of Trustees.
\end{abstract}

Keywords: Total shoulder arthroplasty; reverse prosthesis; impingement

This is a Basic Science Study for which IRB/Ethical Committee approval is not required.

*Reprint requests: Alexandre Terrier, PhD, Laboratory of Biomechanical Orthopedics, Ecole Polytechnique Fédérale de Lausanne, Station 15, CH-1015, Lausanne, Switzerland.

E-mail address: alexandre.terrier@epfl.ch (A. Terrier).
Reverse shoulder arthroplasty (RSA) is currently an accepted treatment for rotator cuff deficient shoulder arthropathy, ${ }^{2}$ with good short- and mid-term results in terms of mobility and patient satisfaction. ${ }^{7}$ 
With reverse prostheses, patients can recover the function with a missing rotator cuff; but the nonanatomical design of this prosthesis induces a medial displacement of the center of rotation, with consecutive impingements between bone and implants, which eventually limit mobility and might damage bone and/or the prosthesis. This has been observed clinically with the first Grammont designs and glenosphere centered on the glenoid. ${ }^{2}$ Scapular notching has been associated with the impingement between the inferior scapular neck and the medial side of the humeral component. ${ }^{19}$

Although the most frequently reported complication of RSA is the scapular notching, ${ }^{7}$ its impact is controversial. ${ }^{19}$ Recently, a prospective study on 60 patients concluded that patients' subjective impression on their shoulders' stability is not correlating with radiological signs of scapular notching, although long-term clinical parameters are affected. $^{18}$

The impingement issue associated with reverse shoulder prostheses has been already investigated in several cadaveric and numerical studies. Different methods to measure the impingement have been proposed, and different solutions have been proposed so far to limit this problem. Nyffeler et al have tested on a cadaveric model the effect of the inferior-superior position and inclination of the baseplate. ${ }^{16}$ With an artificial bone model, Gutierrez et al have evaluated the effect of the glenosphere diameter, the center of rotation offset, the glenosphere position on the glenoid, and humeral neck-shaft angle on the range of motion (ROM) and inferior scapular impingement. ${ }^{9}$ They have concluded that the lateralization of the glenosphere provided the largest effect on ROM. Another artificial bone model was used to compare the ROM of different glenosphere sizes and positions. ${ }^{3}$ Inferior positioning of the glenosphere was found to increase the adduction angle, while a larger glenosphere increased the adductionabduction ROM. Numerical models were also developed to analyze this problem and help improve the implant design. ${ }^{11,17}$ Measurements were also performed on patients, using computed tomography (CT) images. ${ }^{13}$ As discussed by Lévigne et al, the clinical consequence of impingement and notching are unclear. ${ }^{15}$ They conclude that scapular notching is frequent, generally progresses, and is associated with deterioration of some clinical parameters and radiolucent lines. They, however, found no relationship between scapular notching and pain or Constant-Murley score.

In summary, the impingement in reverse shoulder prostheses was clinically observed in the first series of the Grammont prosthesis, but its occurrence and consequences are not fully clear today. Several biomechanical studies have analyzed the impingement of reverse shoulder prostheses for specific and simple movements, which were not related to activities of daily living. The mobility measure was evaluated on the glenohumeral joint, and not related to the global arm motion and scapular mobility. In addition, the RSA has been reported to be associated with extended motion of the scapula. ${ }^{4,14}$ Therefore, we hypothesized that the scapula can compensate the impingements reported by biomechanical studies. This compensation may have an important clinical effect if the patient can indeed adapt the scapula motion.

Therefore, the goal of this study was to test the hypothesis that, during activities of daily living, the functional mobility of the shoulder can be restored by scapular compensation. To answer this question, we have developed a numerical model based on experimental measurements and analyzed shoulder mobility after different configurations of the Aequalis reverse prosthesis.

\section{Materials and methods}

A 3-dimensional computer model was developed from computer tomography (CT) images of a cadaveric shoulder without any sign of pathology. The CT included the entire scapula and the proximal humerus. The scapula and humerus bones were segmented using Amira (Visage Imaging GmbH, Berlin, Germany). The segmentation provided a cloud of points at the bone surface, which was used to build smooth spline surfaces with Geomagic Studio (Geomagic Inc., Morrisville, NC, USA). The reconstructed bone geometry was then superimposed to the CT slices within Amira to evaluate visually the precision of the reconstruction. The reconstructed scapula and humerus were then imported into the computer-aided design (CAD) software SolidWorks (Dassault Systèmes SolidWorks Corp., Concord, MA, USA) to perform the arthroplasty virtually.

Four variations of the reverse shoulder prosthesis Aequalis (Tornier, Edina, MN, USA) were inserted into the virtual shoulder (Fig. 1): the standard 36-mm glenosphere (STD-RSA); the 42-mm glenosphere (LRG-RSA); the lateralized 36-mm glenosphere (LAT-RSA); and the Bony Increased-Offset (BIO-RSA). ${ }^{1}$ For all configurations, the 29-mm glenoid baseplate was positioned at the lower edge of the scapular neck, as usually recommended. All humeral components had a cut-shaft angle of $155^{\circ}$ and were positioned with the recommended retroversion of $20^{\circ}$. The LRGRSA was positioned in the same way as the STD-RSA, but the glenosphere was $42 \mathrm{~mm}$ in diameter. In this case, the corresponding humeral component was used. The LAT-RSA was positioned in the same way as the STD-RSA, but the was glenosphere was lateralized by $10 \mathrm{~mm}$. The BIO-RSA was based on the same design as the STD-RSA, but the baseplate was tilted downward by $10^{\circ}$ and lateralized by a $10-\mathrm{mm}$ bone graft, as recommended by Boileau et al. ${ }^{1}$ The reaming of the scapula and humerus were done in the virtual model, according to the recommended surgical technique. A senior shoulder surgeon (A.F.) performed the 4 implantations in the virtual model.

Four activities of daily living were evaluated: (M1) hand to contra lateral shoulder; (M2) hand to mouth; (M3) combing hair; and (M4) hand to back pocket. For these 4 movements, we simulated the associated motion of the scapula and humerus in the CAD models for the 4 prostheses. The motion of the scapula and humerus was obtained from averaged measurements on 10 healthy volunteers. ${ }^{20}$ This experimental study provided the orientation of the humerus and the scapulae at 100 (equally spaced) time points during these 4 movements. Local coordinate systems and rotations were defined in the same way in the computer model as in the 
STD-RSA 36

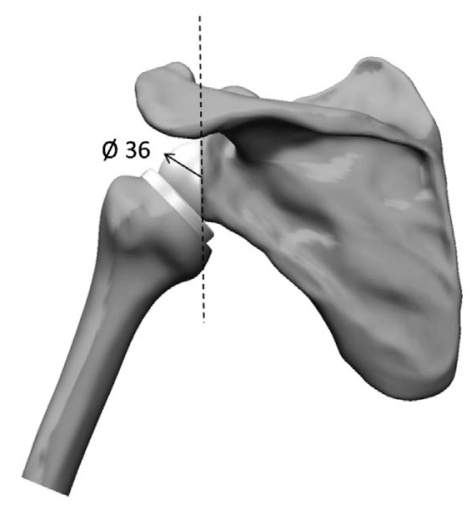

LAT-RSA

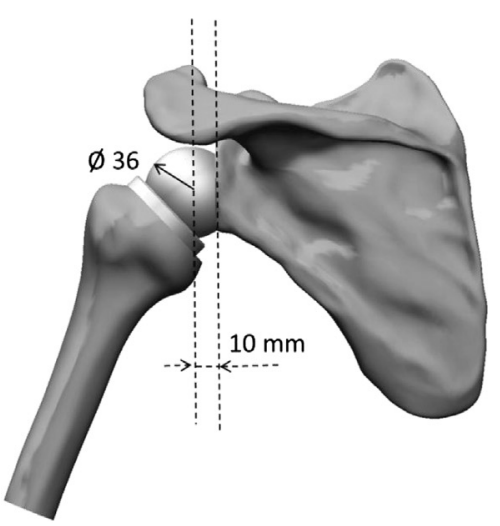

STD-RSA 42

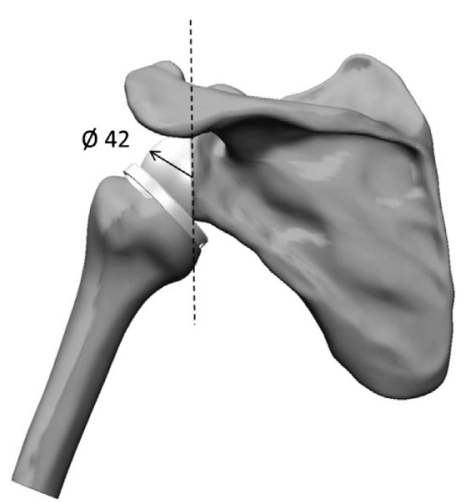

BIO-RSA

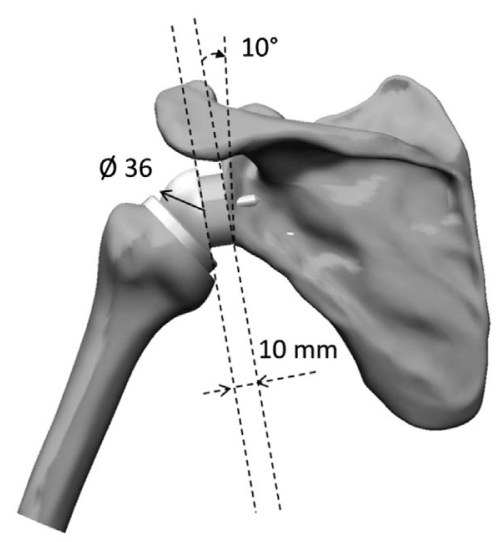

Figure 1 The 4-tested RSA configurations.

experimental study, ${ }^{20}$ according to the ISB recommendations. ${ }^{21}$ As the CT images did not contain the humerus epicondyles, we estimated the humerus $\mathrm{Y}$ axis by a best fit of intramedullary canal and the humerus $\mathrm{X}$ axis by assuming a humeral head retroversion of $20^{\circ} .{ }^{12}$ For the combing hair movement (M3), we only considered the first half of the original measurement, since it was a back and forth movement. Each of the 4 movements was divided in 3 equal parts (of 33 time increments): start, middle, and end. The 100 experimentally measured orientations were reproduced incrementally and manually (by setting the angle values in the associated fields) in the virtual model. The "Interference Detection Tool" of SolidWorks was used to detect any impingement between the scapula, humerus, and components of the prosthesis.

The occurrence of impingement was measured as a percentage of motion free of impingement relatively to the entire movement. A value of $100 \%$ would mean that the "average healthy volunteer" performs the entire movement without any impingement, while a value of $50 \%$ would mean that half of the movement induces impingement. This measure was done for each of the 3 parts of the movement.

The level of impingement was measured by the amplitude of scapular compensation required to avoid this impingement. This scapular compensation was evaluated by incremental rotations of $0.1^{\circ}$ in the 3 orthogonal axes of rotation: pro/retraction, lateral/ medial rotation, anterior/posterior tilt (Fig. 2). Each rotation angle of the scapula was measured independently, by maintaining the other 2 fixed.
In addition, we measured the maximal (free of impingement) range of glenohumeral adduction/abduction in the plane of the scapula. This range was characterized by the maximal adduction and the maximal abduction, using the ISB recommendation.

\section{Results}

There was no impingement of any of the 4 reverse prostheses for 2 of the 4 movements: hand to mouth (M2) and combing hair (M4). For the movement of hand to contralateral shoulder (M1), impingement was only observed in the first part of the movement, which corresponded to the rest position in neutral rotation and elbow to body (Fig. 3). The most important impingement was observed during the third part of the back-pocket movement (M4).

The above 2 situations of impingement occurred only for the STD-RSA and LRG-RSA configurations. Increasing the glenosphere size (LRG-RSA) only partly improved the standard configuration (STD-RSA). The lateralization of the rotation center with an eccentric glenosphere (LAT-RSA) or a bone graft (BIO-RSA) avoided these impingements.

To avoid impingement with the STD-RSA in the rest position (neutral rotation and elbow to body), the scapular compensation angles were $11^{\circ}$ retraction, $7^{\circ}$ medial rotation, 


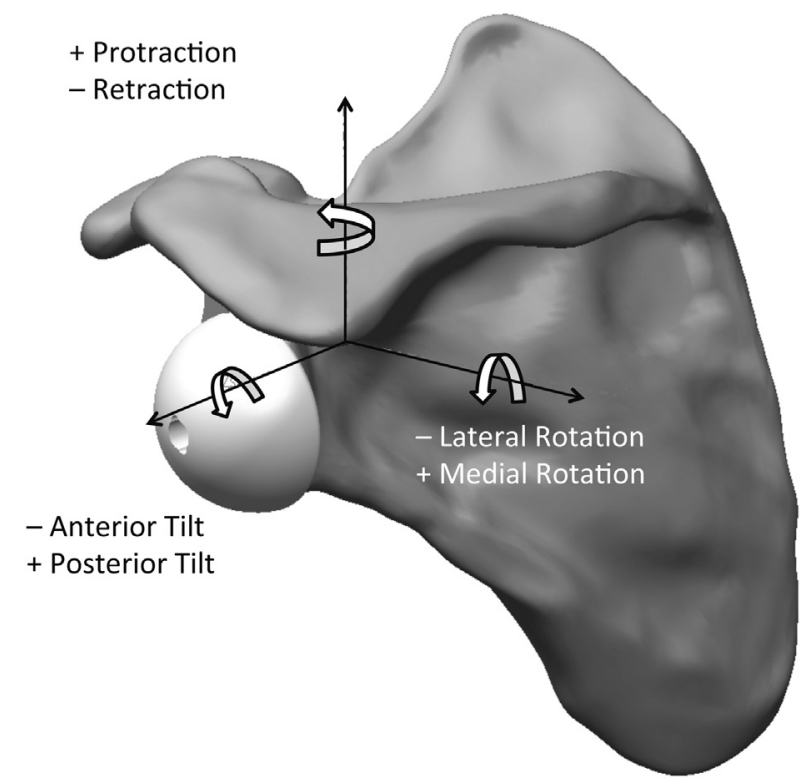

Figure 2 The 3 rotation angles of the scapula.

and $13^{\circ}$ anterior tilt. With the LRG-RSA, they reduced to $5^{\circ}$, $3^{\circ}$, and $7^{\circ}$ in the same directions. To avoid impingement with the STD-RSA at the end of the back-pocket movement, the scapular compensation angles were $8^{\circ}$ retraction, $7^{\circ}$ medial rotation, and $9^{\circ}$ anterior tilt. With the LRG-RSA, they reduced to $3^{\circ}, 3^{\circ}$, and $4^{\circ}$, in the same directions (Fig. 4).

The maximal glenohumeral adduction/abduction angles were $3.6^{\circ} / 88.9^{\circ}$ for the STD-RSA, $2.2^{\circ} / 88.3^{\circ}$ for the LRGRSA, $6.7^{\circ} / 93.4^{\circ}$ for the LAT-RSA, and $16.3^{\circ} / 97.8^{\circ}$ for the BIO-RSA.

\section{Discussion}

Reverse shoulder arthroplasty is an accepted treatment for glenohumeral arthritis associated with irreparable rotator cuff tears. Although major problems of initial reverse designs and positioning have been solved, ${ }^{2,16}$ impingement causing a limitation of mobility is still an open question. ${ }^{2,6,8,15,16} \mathrm{It}$ has been analyzed with different prosthesis design by several biomechanical models ${ }^{8-10,16}$; but it was never evaluated in activities of daily living, and never related to scapular compensation. In the present study, the occurrence of impingement in typical activities of daily living was rather low, and only observed for adducted positions. Besides, the required scapular compensation angles were within the standard deviation of the measurements. Although these results were obtained with a single healthy shoulder and average movements of healthy volunteers, we assume that scapular compensation partly avoids impingement after RSA.

For the tested movements, impingement only occurred between the humerus and the inferior part of the glenoid, when the arm was in an inferior position. When impingement occurred, the scapular compensation angles were below or at the same level as the standard deviations of the kinematics measurements on the healthy volunteers. ${ }^{20}$ Because the starting position was not the same for the 4 activities of daily life, the resting position impingement only occurred for M1. We can, however, estimate that scapular compensation through patient adaptation might reduce the initial impingement and increase the mobility. A larger glenosphere (42 vs. $36 \mathrm{~mm}$ ) provided a slight improvement, while lateralized $(10 \mathrm{~mm})$ glenosphere avoided this inferior impingement. The measured angle of glenohumeral adduction/abduction can be related to a humero-thoracic angle. A value of zero adduction/abduction corresponds approximately to the rest position of the arm. Therefore, we can estimate that the STD-RSA and the LRG-RSA were close to inferior impingement at the rest position, while the LATRSA, and even more the BIO-RSA, provided some mobility in adduction below the rest position. This confirms the results obtained with the 4 activities of daily living. For comparison, the maximum adduction with an anatomical prosthesis was evaluated at $23^{\circ}$ with the same model. The maximal abduction was nearly the same for the STD-RSA and LRG-RSA, but it was improved by nearly $10^{\circ}$ with the LAT-RSA and BIO-RSA.

In a cadaveric study, Nyffeler et al measured the maximal adduction and abduction in several elevation planes, for different positions and inclination of the glenoid baseplate. ${ }^{16}$ For the case that best corresponds to our STD-RSA, the average range of abduction in the scapular plane was $82^{\circ}$. This value is in the same order than the value obtained in our model. With an artificial scapula and humerus, Gutierrez et al measured the difference in abduction angle for 7 configurations of the RSA. ${ }^{10}$ They observed an increase of the range of abduction with a lateralization of the glenosphere up to $97^{\circ}$. Using a computer version of the initial experimental model, they evaluated the abduction ROM in the scapular plane, for various design parameters of the reverse prosthesis. ${ }^{8,9}$ The ROM was about $74^{\circ}$ for the case that best corresponds to the STD-RSA. A glenosphere of $42 \mathrm{~mm}$, instead of $36 \mathrm{~mm}$, increased the ROM by about $6^{\circ}$. A lateralization of $10 \mathrm{~mm}$ increased the ROM by about $30^{\circ}$. The same numerical model predicted that the lateralization of the rotation center was the most important design parameter to increase the glenohumeral abduction. Their results are consistent with our observations. In a 2-dimensional computer model based on 200 scapulae, 6 design features of the RSA were tested to evaluate the optimum gain in adduction. ${ }^{5}$ Although the results are difficult to compare with ours, they also reported an increased adduction with a lateralization and size increase of the glenosphere. The present paper also confirms a clinical study, which reports a reduction in the scapular notching of the lateralized BIO-RSA compared to STD-RSA. ${ }^{1}$

The major strength and novelty of this study was to evaluate the compensation of the scapula to avoid impingements after RSA during activities of daily living. Conversely to all other biomechanical studies, which focus 


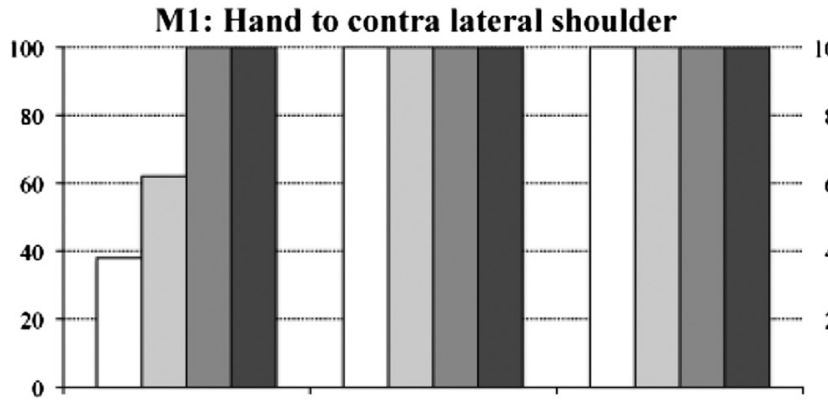

Pl

P2

P3

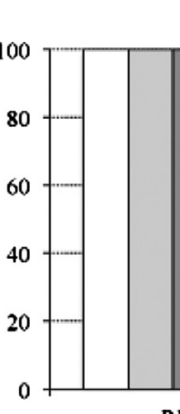

M2: Hand to mouth

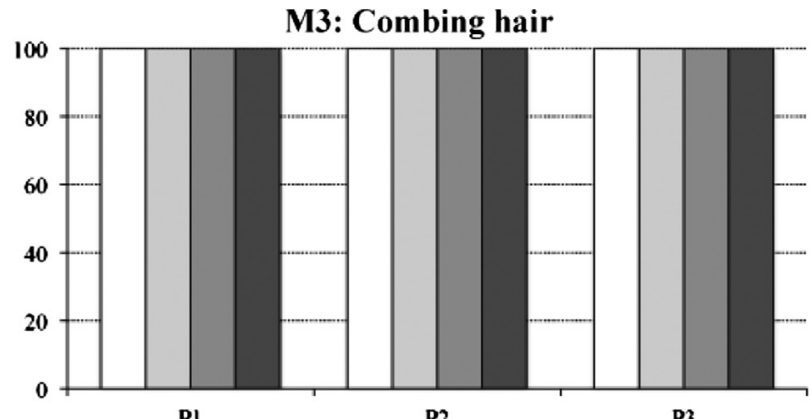

Pl

P2

QSTD-RSA

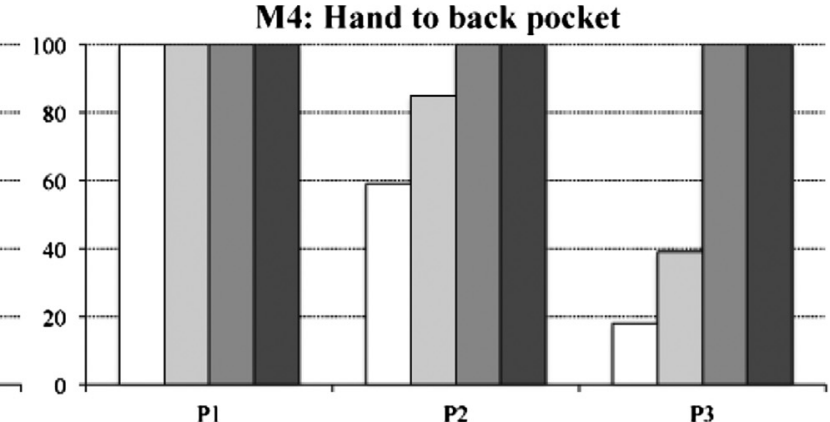

Figure 3 The percentage (relative to healthy shoulders) of possible movement without impingement presented for the 4 movements (M1, M2, M3, M4), divided into 3 parts (start: P1, middle: P2, end: P3) and the 4 RSA configurations (STD-RSA, LRG-RSA, LAT-RSA, BIO-RSA).
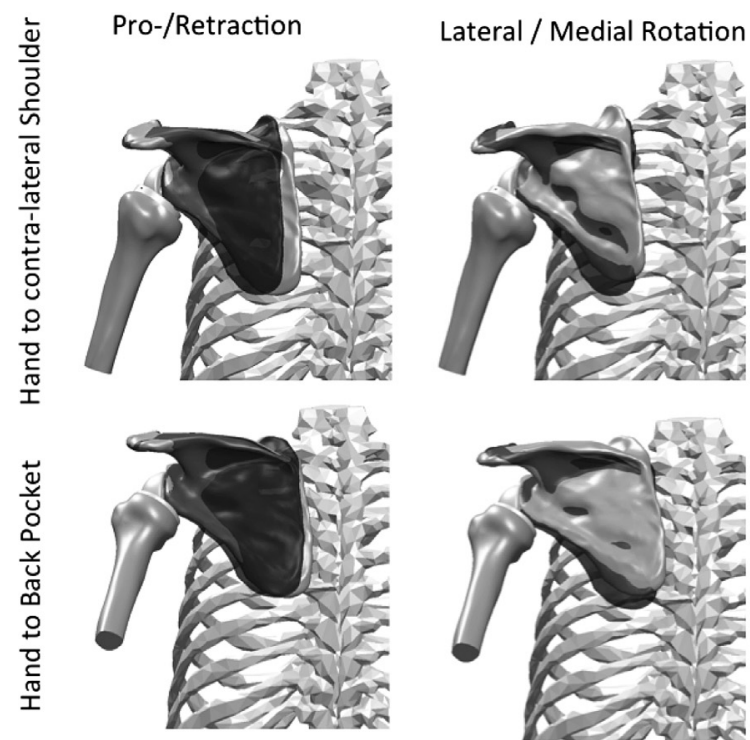

\section{Anterior / Posterior Tilt}
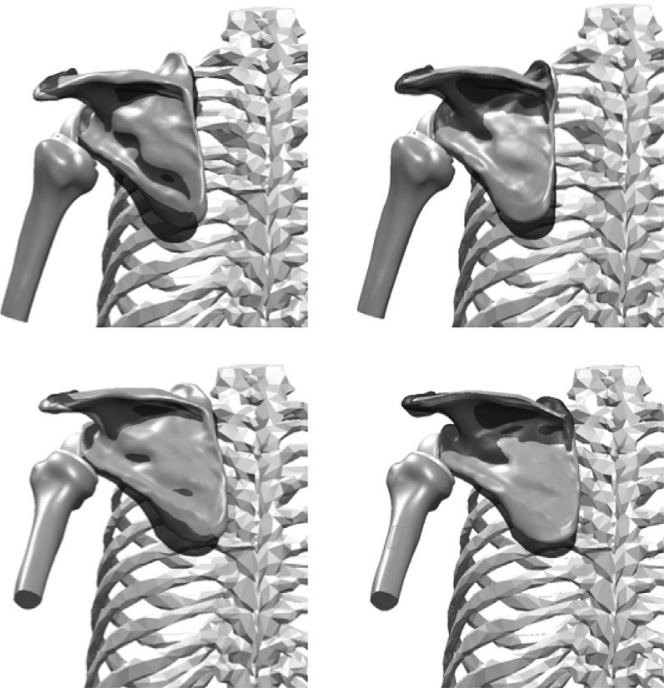

Figure 4 The 2 impingement configurations (rest position and back-pocket) of the STD-RSA showing the compensated position of the scapula in the 3 rotation axes (light grey) and its average natural position (dark grey).

on the maximal range of glenohumeral abduction in a specific plane of elevation, we associated the impingement problem of reverse shoulder prostheses with typical activities from measured kinematics of the humerus and scapula relative to the thorax. As these data were obtained from healthy volunteers, the average motion used here can be understood as the typical movement of a person free of any pathology. This is clearly not the case of the patients, just before and after the surgery. We may still assume that this average healthy movement is representative of the movement that would naturally be perform these patients after a recovery period after the surgery. As in most biomechanical studies, we performed this analysis with only one shoulder, free of any pathology. We could get interesting 
statistical information by doing the same analysis on a cohort of patients with a reverse shoulder prosthesis, and compare our predictions with their clinical score. This would require a geometric reconstruction of the scapula and humerus, which is not always possible from a clinical CT. However, we assume that this limitation to 1 normal shoulder would not change the conclusions of this study. In addition, this study is limited to 4 movements, which do not fully represent all activities of daily living.

\section{Conclusion}

Scapular compensation may reduce impingement associated with reverse shoulder arthroplasty, by transferring the lost gleno-humeral mobility to the scapula-thoracic joint. This mechanism could however induce additional loading of the scapulo-thoracic joint and result in a posterior discomfort in some cases. Further specific clinical attention is necessary to confirm this hypothesis.

\section{Acknowledgments}

This study was partly funded by Tornier (Tornier, Inc., Edina, MN, USA) and by the Center of Translational Biomechanics EPFL (Ecole Polytechnique Fédérale de Lausanne)-CHUV (Centre Hospitalier Universitaire Vaudois, Lausanne)-DAL (Département de l'Appareil Locomoteur, CHUV).

\section{References}

1. Boileau P, Moineau G, Roussanne Y, O'Shea K. Bony increased-offset reversed shoulder arthroplasty: minimizing scapular impingement while maximizing glenoid fixation. Clin Orthop Relat Res 2011;469: 2558-67. http://dx.doi.org/10.1007/s11999-011-1775-4

2. Boileau P, Watkinson DJ, Hatzidakis AM, Balg F. Grammont reverse prosthesis: design, rationale, and biomechanics. J Shoulder Elbow Surg 2005;14:147S-61S. http://dx.doi.org/10.1016/j.jse.2004.10.006

3. Chou J, Malak SF, Anderson IA, Astley T, Poon PC. Biomechanical evaluation of different designs of glenospheres in the SMR reverse total shoulder prosthesis: range of motion and risk of scapular notching. J Shoulder Elbow Surg 2009;18:354-9. http://dx.doi.org/10. 1016/j.jse.2009.01.015

4. de Toledo JM, Loss JF, Janssen TW, van der Scheer JW, Alta TD, Willems WJ, et al. Kinematic evaluation of patients with total and reverse shoulder arthroplasty during rehabilitation exercises with different loads. Clin Biomech (Bristol, Avon) 2012;27:793-800. http:// dx.doi.org/10.1016/j.clinbiomech.2012.04.009

5. de Wilde LF, Poncet D, Middernacht B, Ekelund A. Prosthetic overhang is the most effective way to prevent scapular conflict in a reverse total shoulder prosthesis. Acta Orthop 2010;81:719-26. http://dx.doi. org/10.3109/17453674.2010.538354

6. Edwards TB, Trappey GJ, Riley C, O'Connor DP, Elkousy HA, Gartsman GM. Inferior tilt of the glenoid component does not decrease scapular notching in reverse shoulder arthroplasty: results of a prospective randomized study. J Shoulder Elbow Surg 2012;21: 641-6. http://dx.doi.org/10.1016/j.jse.2011.08.057

7. Farshad M, Gerber C. Reverse total shoulder arthroplasty-from the most to the least common complication. Int Orthop 2010;34:1075-82. http://dx.doi.org/10.1007/s00264-010-1125-2

8. Gutierrez S, Comiskey CA, Luo Z-P, Pupello DR, Frankle MA. Range of impingement-free abduction and adduction deficit after reverse shoulder arthroplasty. Hierarchy of surgical and implant-designrelated factors. J Bone Joint Surg (Am) 2008;90:2606-15. http://dx. doi.org/10.2106/JBJS.H.00012

9. Gutierrez S, Levy JC, Frankle MA, Cuff D, Keller TS, Pupello DR, et al. Evaluation of abduction range of motion and avoidance of inferior scapular impingement in a reverse shoulder model. J Shoulder Elbow Surg 2008;17:608-15. http://dx.doi.org/10.1016/j.jse. 2007.11.010

10. Gutierrez S, Levy JC, Lee WE III, Keller TS, Maitland ME. Center of rotation affects abduction range of motion of reverse shoulder arthroplasty. Clin Orthop Relat Res 2007;458:78-82. http://dx.doi.org/ 10.1097/BLO.0b013e31803d0f57

11. Gutierrez S, Luo ZP, Levy J, Frankle MA. Arc of motion and socket depth in reverse shoulder implants. Clin Biomech 2009;24:473-9. http://dx.doi.org/10.1016/j.clinbiomech.2009.02.008

12. Harrold F, Wigderowitz C. A three-dimensional analysis of humeral head retroversion. J Shoulder Elbow Surg 2012;21:612-7. http://dx. doi.org/10.1016/j.jse.2011.04.005

13. Karelse AT, Bhatia DN, De Wilde LF. Prosthetic component relationship of the reverse Delta III total shoulder prosthesis in the transverse plane of the body. J Shoulder Elbow Surg 2008;17:602-7. http://dx.doi.org/10.1016/j.jse.2008.02.005

14. Kontaxis A, Johnson GR. Adaptation of scapula lateral rotation after reverse anatomy shoulder replacement. Comput Meth Biomech Biomed Eng 2008;11:73-80. http://dx.doi.org/10.1080/10255840802296590

15. Levigne C, Garret J, Boileau P, Alami G, Favard L, Walch G. Scapular notching in reverse shoulder arthroplasty: is it important to avoid it and how? Clinical orthopaedics and related research 2011;469:251220. http://dx.doi.org/10.1007/s11999-010-1695-8

16. Nyffeler RW, Werner CM, Gerber C. Biomechanical relevance of glenoid component positioning in the reverse Delta III total shoulder prosthesis. J Shoulder Elbow Surg 2005;14:524-8. http://dx.doi.org/10. 1016/j.jse.2004.09.010

17. Roche C, Flurin PH, Wright T, Crosby LA, Mauldin M, Zuckerman JD. An evaluation of the relationships between reverse shoulder design parameters and range of motion, impingement, and stability. J Shoulder Elbow Surg 2009;18:734-41. http://dx.doi.org/10. 1016/j.jse.2008.12.008

18. Sadoghi P, Leithner A, Vavken P, Holzer A, Hochreiter J, Weber G, et al. Infraglenoidal scapular notching in reverse total shoulder replacement: a prospective series of 60 cases and systematic review of the literature. BMC musculoskeletal disorders 2011;12:101. http://dx. doi.org/10.1186/1471-2474-12-101

19. Sirveaux F, Favard L, Oudet D, Huquet D, Walch G, Mole D. Grammont inverted total shoulder arthroplasty in the treatment of glenohumeral osteoarthritis with massive rupture of the cuff. Results of a multicentre study of 80 shoulders. J Bone Joint Surg Br 2004;86: 388-95. http://dx.doi.org/10.1302/0301-620x.86b3.14024

20. van Andel CJ, Wolterbeek N, Doorenbosch CA, Veeger DH, Harlaar J. Complete 3D kinematics of upper extremity functional tasks. Gait Posture 2008;27:120-7. http://dx.doi.org/10.1016/j.gaitpost.2007. 03.002

21. Wu G, van der Helm FC, Veeger HE, Makhsous M, Van Roy P, Anglin C, et al. ISB recommendation on definitions of joint coordinate systems of various joints for the reporting of human joint motion-Part II: shoulder, elbow, wrist and hand. J Biomech 2005;38:981-92. http:// dx.doi.org/10.1016/J.Jbiomech.2004.05.042 